\title{
A note on stability in three-phase-lag heat conduction
}

\author{
RAMÓN QUINTANILLA ${ }^{1}$ \\ Department of Applied Mathematics II \\ UPC Terrassa, Colom 11, 08222 Terrassa, Spain \\ ramon.quintanilla@upc.edu \\ REINHARD RACKE ${ }^{2}$ \\ Department of Mathematics and Statistics \\ University of Konstanz \\ 78457 Konstanz, Germany \\ reinhard.racke@uni-konstanz.de
}

Abstract: In this note we consider two cases in the theory of the heat conduction models with three-phase-lag. For each one we propose a suitable Lyapunov function. These functions are relevant tools which allow to study several qualitative properties. We obtain conditions on the material parameters to guarantee the exponential stability of solutions. The spectral analysis complements the results and we show that if the conditions obtained to prove the exponential stability are not satisfied, then we can obtain the instability of solutions for suitable domains. We believe that this kind of results is fundamental to clarify the applicability of the models.

\section{Introduction}

There are several parabolic and hyperbolic theories which describe the heat conduction, the latter also being called theories of second sound, where the propagation of heat is modeled with finite propagation speed, in contrast to the classical model using Fourier's law leading to infinite propagation speed of heat signals, see the survey by Chandrasekharaiah [1] or the book of Müller and Ruggeri [3]. Recently, there have been considered the dual-phase-lag heat equations which were proposed by Tzou $[9,10]$ and investigated by Quintanilla and Racke $[4,5,6,7]$ and Wang et al. [11, 12, 13]. Here we consider the threephase-lag heat conduction recently proposed by Roy [8]. In this paper, several types of heat equations were considered. However we focus our attention to the two new equations

\footnotetext{
${ }^{0}$ AMS subject classification: 35 L 35, 80 A 20

Keywords and phrases: hyperbolic models in heat conduction, stability

${ }^{1}$ Supported by the project "Qualitative study of thermomechanical problems" (MTM2006-03706)

${ }^{2}$ Supported by the DFG-project "Hyperbolic Thermoelasticity" (RA 504/3-2).
} 
which are the following:

$$
\rho c_{\nu} \ddot{T}+\tau_{q} \rho c_{\nu} \dddot{T}=k^{*} \triangle T+\tau_{\nu}^{*} \triangle \dot{T}+k \tau_{T} \triangle \ddot{T}
$$

and

$$
\rho c_{\nu} \ddot{T}+\tau_{q} \rho c_{\nu} \dddot{T}+\frac{\tau_{q}^{2}}{2} \rho c_{\nu} \dddot{T}=k^{*} \triangle T+\tau_{\nu}^{*} \triangle \dot{T}+k \tau_{T} \triangle \ddot{T} .
$$

where the coefficients appearing are positive, constant material parameters.

We observe that equation (1.2) is of wave equation type for $\ddot{T}$,

$$
\frac{\partial^{2}}{\partial t^{2}}(\ddot{T})-\frac{2 k \tau_{T}}{\tau_{q}^{2} \rho c_{\nu}} \Delta \ddot{T}=\text { l.o.t. }
$$

with lower order terms on the right-hand side. The characteristic wave speeds are

$$
s_{1,2}= \pm \sqrt{\frac{2 k \tau_{T}}{\tau_{q}^{2} \rho c_{\nu}}}
$$

Actually, in one space dimension, we can reformulate (1.2) as first-order system for

$$
\begin{gathered}
V:=\left(T, \dot{T}, \ddot{T}, \dddot{T}, T_{x}, \dot{T}_{x}, \ddot{T}_{x}\right)^{\prime} \\
\dot{V}+A V_{x}+B V=0
\end{gathered}
$$

with

$$
A=\left(\begin{array}{ccccccc}
0 & 0 & 0 & 0 & 0 & 0 & 0 \\
0 & 0 & 0 & 0 & 0 & 0 & 0 \\
0 & 0 & 0 & 0 & 0 & 0 & 0 \\
0 & 0 & 0 & 0 & -\frac{2 k^{*}}{\tau_{q}^{2} \rho c_{\nu}} & -\frac{2 \tau_{\nu}^{*}}{\tau_{q}^{2} \rho c_{\nu}} & -\frac{2 k \tau_{T}}{\tau_{q}^{2} \rho c_{\nu}} \\
0 & -1 & 0 & 0 & 0 & 0 & 0 \\
0 & 0 & -1 & 0 & 0 & 0 & 0 \\
0 & 0 & 0 & -1 & 0 & 0 & 0
\end{array}\right), B=\left(\begin{array}{ccccccc}
0 & -1 & 0 & 0 & 0 & 0 & 0 \\
0 & 0 & -1 & 0 & 0 & 0 & 0 \\
0 & 0 & 0 & -1 & 0 & 0 & 0 \\
0 & 0 & \frac{2}{\tau_{q}^{2}} & \frac{2}{\tau_{q}} & 0 & 0 & 0 \\
0 & 0 & 0 & 0 & 0 & 0 & 0 \\
0 & 0 & 0 & 0 & 0 & 0 & 0 \\
0 & 0 & 0 & 0 & 0 & 0 & 0
\end{array}\right) .
$$

The eigenvalues $\gamma_{1-7}$ of $A$ are all real,

$$
\gamma_{1}=\gamma_{2}=\gamma_{3}=\gamma_{4}=\gamma_{5}=0, \quad \gamma_{6,7}= \pm \sqrt{\frac{2 k \tau_{T}}{\tau_{q}^{2} \rho c_{\nu}}}
$$

Thus, it is a hyperbolic system with finite propagation speed, and we recover with $\gamma_{6,7}$ the characteristic wave speeds $s_{1,2}$.

The equation (1.1) is of heat equation, i.e. parabolic, type for $\ddot{T}$,

$$
\frac{\partial}{\partial t}(\ddot{T})-\frac{k \tau_{T}}{\tau_{q} \rho c_{\nu}} \Delta \ddot{T}=\text { l.o.t. }
$$


hence infinite propagation speed is expected.

In this paper we propose a Lyapunov function for each equation (1.1) and (1.2). These functions will be a powerful tool to study the qualitative aspects of the solutions of these equations. We also consider the spectral analysis of these equations. Both analysis aspects characterize suitable conditions on the constitutive parameters to guarantee the stability or the instability of solutions, respectively.

We believe that this kind of results is fundamental to clarify the applicability of the models. In fact, if a model does not guarantee the stability of solutions, it can not be a good candidate to describe the heat conduction. For the exponential stability we remark that there are examples for which Fourier's law of heat conduction leads to exponential stability, while Cattaneo's law does not, cp. [2]. Thus, we believe that our results are the first step to clarify before to develop any qualitative study in these theories.

The paper is organized as follows: In Section 2 we prove (exponential) stability for the equation (1.1) whenever condition (2.1) holds. A relevant tool, a Lyapunov function, is proposed. In Section 3, we get the instability of solutions whenever (2.1) fails. In the same manner, sections 4 and 5 are devoted to the discussion of equation (1.2) with respect to the condition (4.1). In Section 6, we summarize our results.

\section{Stability of solutions: Equation (1.1)}

In this section we will prove the stability of solutions of equation (1.1) whenever we assume that the constitutive constants satisfy:

$$
\tau_{\nu}^{*}>\tau_{q} k^{*}
$$

We will consider the problem determined by the equation (1.1) in a bounded domain $B$ smooth enough to guarantee the use of the divergence theorem. To determine a well posed problem we impose the initial conditions:

$$
T(\mathbf{x}, 0)=T_{0}(\mathbf{x}), \quad \dot{T}(\mathbf{x}, 0)=\theta_{0}(\mathbf{x}), \quad \ddot{T}(\mathbf{x}, 0)=\eta_{0}(\mathbf{x}), \quad \mathbf{x} \in B,
$$

and the homogeneous boundary conditions

$$
T(\mathbf{x}, t)=0, \quad \mathbf{x} \in \partial B
$$

Now, we will prove the exponential decay of solutions of the problem determined by equation (1.1), the initial conditions (2.2) and the boundary conditions (2.3). We define an energy functional which is a useful tool to study our problem:

$$
F(t):=\frac{1}{2} \int_{B}\left(\rho c_{\nu}\left(\dot{T}+\tau_{q} \ddot{T}\right)^{2}+k^{*}\left|\nabla\left(T+\tau_{q} \dot{T}\right)\right|^{2}+\left(\tau_{q}\left(\tau_{\nu}^{*}-k^{*} \tau_{q}\right)+k \tau_{T}\right)|\nabla \dot{T}|^{2}\right) d v
$$


We have

$$
\begin{array}{r}
F^{\prime}(t)=-\int_{B} \nabla\left(\dot{T}+\tau_{q} \ddot{T}\right) \cdot\left(\left(\tau_{\nu}^{*}-k^{*} \tau_{q}\right) \nabla \dot{T}+k \tau_{T} \nabla \ddot{T}\right) d v+ \\
+\left(\tau_{q}\left(\tau_{\nu}^{*}-k^{*} \tau_{q}\right)+k \tau_{T}\right) \int_{B} \nabla \dot{T} . \nabla \ddot{T} d v .
\end{array}
$$

Thus, we obtain

$$
F^{\prime}(t)=-\int_{B}\left(\left(\tau_{\nu}^{*}-k^{*} \tau_{q}\right)|\nabla \dot{T}|^{2}+k \tau_{T} \tau_{q}|\nabla \ddot{T}|^{2}\right) d v \leq 0 .
$$

This inequality shows that the solutions of our problem are stable whenever we assume that the constitutive coefficients satisfy the condition (2.1). However, we are going to see a stronger result We will prove that the solutions decay in an exponential way.

First, we note that the function $F$ defines an energy of the type of $|\nabla T|^{2},|\nabla \dot{T}|^{2},|\ddot{T}|^{2}$. Now, we define the function

$$
G(t):=\int_{B}\left(\frac{\tau_{\nu}^{*}}{2}|\nabla T|^{2}+k \tau_{T} \nabla T . \nabla \dot{T}+\rho c_{\nu} T \dot{T}+\rho c_{\nu} \tau_{q} T \ddot{T}\right) d v .
$$

We have

$$
G^{\prime}(t)=-k^{*} \int_{B}|\nabla T|^{2} d v+\int_{B}\left(k \tau_{T}|\nabla \dot{T}|^{2}+\rho c_{\nu} \dot{T}^{2}+\rho c_{\nu} \tau_{q} \dot{T} \ddot{T}\right) d v
$$

Now, it is clear that if we take $\epsilon$ small enough we can guarantee that $F(t)+\epsilon G(t)$ defines a equivalent norm to $F(t)$ and such that

$$
\int_{B}\left(\left(\tau_{\nu}^{*}-k^{*} \tau_{q}\right)|\nabla \dot{T}|^{2}+k \tau_{T} \tau_{q}|\nabla \ddot{T}|^{2}\right) d v-\epsilon \int_{B}\left(k \tau_{T}|\nabla \dot{T}|^{2}+\rho c_{\nu} \dot{T}^{2}+\rho c_{\nu} \tau_{q} \dot{T} \ddot{T}\right) d v
$$

is equivalent to an energy of the type of

$$
\int_{B}\left(|\nabla \dot{T}|^{2}+|\nabla \ddot{T}|^{2}\right) d v
$$

Thus, it is clear that

$$
F^{\prime}(t)+\epsilon G^{\prime}(t) \leq-\kappa \int_{B}\left(|\nabla T|^{2}+|\nabla \dot{T}|^{2}+|\ddot{T}|^{2}\right) d v \leq-\kappa^{*}(F(t)+\epsilon G(t))
$$

where $\kappa$ and $\kappa^{*}$ are two calculable positive constants. Thus, it follows the existence of two positive constants $C$ and $\vartheta$ such that

$$
F(t) \leq C F(0) \exp (-\vartheta t)
$$

for all $t \geq 0$. This inequality says that the solutions decay in an exponential way. 


\section{Spectral analysis for (1.1)}

We have proved that whenever we assume that inequality (2.1) holds, then the solutions decay in a exponential way. In this section we will see that whenever this condition fails, then there exist domains $B$ such that the problem has unstable solutions. To this end, let us consider functions of the form

$$
T(x, t)=\exp (\omega t) \Phi(x)
$$

where $\Phi$ satisfies

$$
\triangle \Phi+\lambda \Phi=0 \text { in } B \text { and } \Phi=0 \text { on } \partial B .
$$

If the function (3.1) is a solution of (1.1) $\omega$ must satisfy the equation

$$
\tau_{q} \rho c_{\nu} x^{3}+\left(\rho c_{\nu}+k \tau_{T} \lambda_{n}\right) x^{2}+\tau_{\nu}^{*} \lambda_{n} x+k^{*} \lambda_{n}=0
$$

where $\left(\lambda_{n}\right)_{n}$ denote the eigenvalues of the negative Laplace operator for Dirichlet boundary conditions.

By the Hurwitz criterion, we know that all three roots of the polynomial

$$
\beta^{3}+l_{1} \beta^{2}+l_{2} \beta+l_{3}=0
$$

have negative real parts if and only if

$$
l_{j}>0, j=1,2,3, \quad l_{1} l_{2}>l_{3}
$$

holds. For (3.2) this conditions turns into

$$
\left(\rho c_{\nu}+k \tau_{T} \lambda_{n}\right) \tau_{\nu}^{*}>\tau_{q} \rho c_{\nu} k^{*}
$$

If this condition shall be satisfied uniformly in $\lambda_{n}$ then we need that (2.1) holds. In fact, we can extend this condition a little bit. Let the eigenvalues be arranged increasingly with $\lambda_{1}$ denoting the smallest eigenvalue. Then, for the stability, it is sufficient that

$$
\lambda_{1}>\frac{\left(\tau_{q} k^{*}-\tau_{\nu}^{*}\right) \rho c_{\nu}}{k \tau_{T}}
$$

On the other hand if (2.1) does not hold, we can always select a domain in such a way that (3.4) does not hold either and then, there exist unstable solutions.

\section{Stability of solutions: equation (1.2)}

The aim of this section is to prove that the solutions of the problem determined by the equation (1.2), the initial conditions (2.2) and the boundary conditions (2.3) are stable whenever

$$
\frac{2 k \tau_{T}}{\tau_{q}}>\tau_{\nu}^{*}>k^{*} \tau_{q}
$$


holds.

Our approach will be similar to the one used in section 2 for equation (1.1). However, here the problem has a greater complexity requiring more sophisticated functionals.

Let us define the energy functional

$$
\begin{gathered}
F(t):=\frac{1}{2} \int_{B}\left(\rho c_{\nu}\left(\dot{T}+\tau_{q} \ddot{T}+\frac{\tau_{q}^{2}}{2} \dddot{T}\right)^{2}+k^{*}\left|\nabla\left(T+\tau_{q} \dot{T}+\frac{\tau_{q}^{2}}{2} \ddot{T}\right)\right|^{2}+\tau_{q}\left(\tau_{\nu}^{*}-k^{*} \tau_{q}\right)\left|\nabla\left(\dot{T}+\frac{\tau_{q}}{2} \ddot{T}\right)\right|^{2}\right. \\
\left.+\left(k \tau_{T}-k^{*} \frac{\tau_{q}^{2}}{2}\right)|\nabla \dot{T}|^{2}+\frac{\tau_{q}^{2}}{2}\left(k \tau_{T}-\frac{\tau_{q} \tau_{\nu}^{*}}{2}\right)|\nabla \ddot{T}|^{2}\right) d v
\end{gathered}
$$

We have

$$
\begin{gathered}
F^{\prime}(t)=-\int_{B}\left(\nabla\left(\dot{T}+\tau_{q} \ddot{T}+\frac{\tau_{q}^{2}}{2} \dddot{T}\right) \cdot\left(\left(\tau_{\nu}^{*}-k^{*} \tau_{q}\right) \nabla \dot{T}+\left(k \tau_{T}-k^{*} \frac{\tau_{q}^{2}}{2}\right) \nabla \ddot{T}\right)\right) d v \\
+\int_{B}\left(\tau_{q}\left(\tau_{\nu}^{*}-k^{*} \tau_{q}\right) \nabla\left(\dot{T}+\frac{\tau_{q}}{2} \ddot{T}\right) \cdot \nabla\left(\ddot{T}+\frac{\tau_{q}}{2} \dddot{T}\right)+\left(k \tau_{T}-k^{*} \frac{\tau_{q}^{2}}{2}\right) \nabla \dot{T} \cdot \nabla \ddot{T}\right) d v \\
+\int_{B}\left(\frac{\tau_{q}^{2}}{2}\left(k \tau_{T}-\frac{\tau_{q} \tau_{\nu}^{*}}{2}\right) \nabla \ddot{T} \cdot \nabla \dddot{T}\right) d v .
\end{gathered}
$$

It follows that the following equalities

$$
F^{\prime}(t)=-\left(\tau_{\nu}^{*}-k^{*} \tau_{q}\right) \int_{B}|\nabla \dot{T}|^{2} d v-\left(\tau_{q}\left(k \tau_{T}-k^{*} \frac{\tau_{q}^{2}}{2}\right)-\frac{\tau_{q}^{2}}{2}\left(\tau_{\nu}^{*}-k^{*} \tau_{q}\right)\right) \int_{B}|\nabla \ddot{T}|^{2} d v
$$

$-\left(k \tau_{T}-k^{*} \frac{\tau_{q}^{2}}{2}\right) \int_{B} \nabla \dot{T} . \nabla \ddot{T} d v-\tau_{q}\left(\tau_{\nu}^{*}-k^{*} \tau_{q}\right) \int_{B} \nabla \ddot{T} . \nabla \dot{T} d v-\frac{\tau_{q}^{2}}{2}\left(\tau_{\nu}^{*}-k^{*} \tau_{q}\right) \int_{B} \nabla \dddot{T} \cdot \nabla \dot{T} d v$ $-\frac{\tau_{q}^{2}}{2}\left(k \tau_{T}-k^{*} \frac{\tau_{q}^{2}}{2}\right) \int_{B} \nabla \dddot{T} \cdot \nabla \ddot{T} d v+\tau_{q}\left(\tau_{\nu}^{*}-k^{*} \tau_{q}\right) \int_{B} \nabla \dot{T} \cdot \nabla \ddot{T} d v+\tau_{q} \frac{\tau_{q}^{2}}{2}\left(\tau_{\nu}^{*}-k^{*} \tau_{q}\right) \int_{B} \nabla \dot{T} \cdot \nabla \dddot{T} d v$ $+\tau_{q} \frac{\tau_{q}^{2}}{4}\left(\tau_{\nu}^{*}-k^{*} \tau_{q}\right) \int_{B} \nabla \ddot{T} . \nabla \dddot{T} d v+\left(k \tau_{T}-k^{*} \frac{\tau_{q}^{2}}{2}\right) \int_{B} \nabla \dot{T} . \nabla \ddot{T} d v+\frac{\tau_{q}^{2}}{2}\left(k \tau_{T}-\frac{\tau_{q} \tau_{\nu}^{*}}{2}\right) \int_{B} \nabla \ddot{T} . \nabla \dddot{T} d v$

$$
\begin{gathered}
=-\left(\tau_{\nu}^{*}-k^{*} \tau_{q}\right) \int_{B}|\nabla \dot{T}|^{2} d v-\tau_{q}\left(k \tau_{T}-\frac{\tau_{q} \tau_{\nu}^{*}}{2}\right) \int_{B}|\nabla \ddot{T}|^{2} d v \\
+\left(-\frac{\tau_{q}^{2}}{2}\left(k \tau_{T}-k^{*} \frac{\tau_{q}^{2}}{2}\right)+\frac{\tau_{q}^{3}}{4}\left(\tau_{\nu}^{*}-k^{*} \tau_{q}\right)+\frac{\tau_{q}^{2}}{2}\left(k \tau_{T}-\frac{\tau_{q} \tau_{\nu}^{*}}{2}\right)\right) \int_{B} \nabla \ddot{T} . \nabla \dddot{T} d v
\end{gathered}
$$

hold. Thus, we obtain

$$
F^{\prime}(t)=-\left(\tau_{\nu}^{*}-k^{*} \tau_{q}\right) \int_{B}|\nabla \dot{T}|^{2} d v-\tau_{q}\left(k \tau_{T}-\frac{\tau_{q} \tau_{\nu}^{*}}{2}\right) \int_{B}|\nabla \ddot{T}|^{2} d v
$$

This inequality shows the stability of solutions whenever conditions (4.1) holds. Again, we want to prove the exponential decay. For this purpose we define the function

$$
G_{1}(t):=\frac{1}{2} \int_{B}\left(\frac{\tau_{q}^{2}}{2} \rho c_{\nu}(\dddot{T})^{2}+\rho c_{\nu}(\ddot{T})^{2}+k \tau_{T}|\nabla \ddot{T}|^{2}+2 \tau_{\nu}^{*} \nabla \dot{T} . \nabla \ddot{T}+2 k^{*} \nabla T . \nabla \ddot{T}\right) d v .
$$


We have

$$
G_{1}^{\prime}(t)=-\tau_{q} \rho c_{\nu} \int_{B}|\dddot{T}|^{2} d v+\int_{B}\left(\tau_{\nu}^{*}|\nabla \ddot{T}|^{2}+k^{*} \nabla \dot{T} \cdot \nabla \ddot{T}\right) d v .
$$

We also define a new function

$$
G_{2}(t):=\int_{B}\left(\frac{\tau_{\nu}^{*}}{2}|\nabla T|^{2}+k \tau_{T} \nabla T . \nabla \dot{T}+\rho c_{\nu} T \dot{T}+\tau_{q} \rho c_{\nu} T \ddot{T}+\frac{\tau_{q}^{2}}{2} \rho c_{\nu} T \dddot{T}\right) d v .
$$

We have

$$
G_{2}^{\prime}(t)=-k^{*} \int_{B}|\nabla T|^{2} d v+\int_{B}\left(k \tau_{T}|\nabla \dot{T}|^{2}+\rho c_{\nu}(\dot{T})^{2}+\tau_{q} \rho c_{\nu} \dot{T} \ddot{T}+\frac{\tau_{q}^{2}}{2} \rho c_{\nu} \dot{T} \dddot{T}\right) d v
$$

If we take $\epsilon_{2}$ small enough we can guarantee that

$$
\epsilon_{2} G_{2}^{\prime}(t)+G_{1}^{\prime}(t) \leq-\epsilon_{2} k^{*} \int_{B}|\nabla T|^{2} d v-\int_{B} \frac{\tau_{q} \rho c_{\nu}}{2}|\dddot{T}|^{2} d v+C^{*} \int_{B}\left(|\nabla \dot{T}|^{2}+|\nabla \ddot{T}|^{2}\right) d v .
$$

Here $C^{*}$ is a computable positive constant.

If we take now $\epsilon_{1}$ small enough, we can guarantee that the function $F(t)+\epsilon_{1}\left(G_{1}(t)+\right.$ $\left.\epsilon_{2} G_{2}(t)\right)$ is equivalent to the function $F(t)$. At the same time we can see that

$$
\begin{gathered}
F^{\prime}(t)+\epsilon_{1}\left(G_{1}^{\prime}(t)+\epsilon_{2} G_{2}^{\prime}(t)\right) \leq-\kappa \int_{B}\left(|\nabla T|^{2}+|\nabla \dot{T}|^{2}+|\nabla \ddot{T}|^{2}+(\dddot{T})^{2}\right) d v \\
-\kappa^{*}\left(F(t)+\epsilon_{1}\left(G_{1}(t)+\epsilon_{2} G_{2}(t)\right)\right) .
\end{gathered}
$$

Again $\kappa$ and $\kappa^{*}$ are calculable positive constants. Thus, we can obtain an estimate similar to (2.7) and the exponential stability of solutions is proved.

\section{$5 \quad$ Spectral analysis for $(1.2)$}

The aim of this section is to prove that whenever condition (4.1) fails then there exist domains $B$ such that unstable solutions of the problem determined by equation (1.2) and the boundary and initial conditions (2.2), (2.3) exist.

If we look for solutions of the form (3.1) to the equation (1.2), $\omega$ must satisfy the equation

$$
\frac{\rho c_{\nu} \tau_{q}^{2}}{2} x^{4}+\rho c_{\nu} \tau_{q} x^{3}+\left(\rho c_{\nu}+k \tau_{T} \lambda_{n}\right) x^{2}+\tau_{\nu}^{*} \lambda_{n} x+k^{*} \lambda_{n}=0
$$

We can write it in the following form

$$
x^{4}+a_{1} x^{3}+a_{2} x^{2}+a_{3} x+a_{4}=0,
$$

where

$$
a_{1}=\frac{2}{\tau_{q}}, \quad a_{2}=\frac{2}{\tau_{q}^{2}}+\frac{2 k \tau_{T} \lambda_{n}}{\tau_{q}^{2} \rho c_{\nu}}, \quad a_{3}=\frac{2 \tau_{\nu}^{*} \lambda_{n}}{\tau_{q}^{2} \rho c_{\nu}}, a_{4}=\frac{2 k^{*} \lambda_{n}}{\tau_{q}^{2} \rho c_{\nu}}
$$


We know that the Hurwitz criterium says that the solutions of the equation have negative real part if and only if all the $a_{i}$ are positive and the following conditions

$$
a_{1} a_{2}-a_{3}>0, \quad a_{1} a_{2} a_{3}-a_{1}^{2} a_{4}-a_{3}^{2}>0
$$

hold. In our case, we have

$$
a_{1} a_{2}-a_{3}=\frac{2}{\tau_{q}}\left(\frac{2}{\tau_{q}^{2}}+\frac{2 k \tau_{T} \lambda_{n}}{\tau_{q}^{2} \rho c_{\nu}}\right)-\frac{2 \tau_{\nu}^{*} \lambda_{n}}{\tau_{q}^{2} \rho c_{\nu}}=\frac{4}{\tau_{q}^{3}}+\frac{4 k \tau_{T}-2 \tau_{q} \tau_{\nu}^{*}}{\tau_{q}^{3} \rho c_{\nu}} \lambda_{n}
$$

To guarantee that this is positive for every $\lambda_{n}$, we need to impose

$$
\tau_{\nu}^{*}<\frac{2 k \tau_{T}}{\tau_{q}}
$$

The second relation is

$$
\begin{gathered}
a_{1} a_{2} a_{3}-a_{1}^{2} a_{4}-a_{3}^{2}=\frac{2}{\tau_{q}}\left(\frac{2}{\tau_{q}^{2}}+\frac{2 k \tau_{T} \lambda_{n}}{\tau_{q}^{2} \rho c_{\nu}}\right) \frac{2 \tau_{\nu}^{*} \lambda_{n}}{\tau_{q}^{2} \rho c_{\nu}}-\left(\frac{2}{\tau_{q}}\right)^{2} \frac{2 k^{*} \lambda_{n}}{\tau_{q}^{2} \rho c_{\nu}}-\left(\frac{2 \tau_{\nu}^{*} \lambda_{n}}{\tau_{q}^{2} \rho c_{\nu}}\right)^{2} \\
=\frac{8 \lambda_{n}}{\tau_{q}^{5} \rho c_{\nu}}\left(\tau_{\nu}^{*}-k^{*} \tau_{q}\right)+\frac{\tau_{\nu}^{*} \lambda_{n}^{2}}{\tau_{q}^{5} \rho^{2} c_{\nu}^{2}}\left(8 k \tau_{T}-4 \tau_{\nu}^{*} \tau_{q}\right) .
\end{gathered}
$$

If we assume that (5.2) holds, the second condition is

$$
k^{*} \tau_{q}<\tau_{\nu}^{*}
$$

If we assume that (5.2) fails, we can always select $\lambda_{n}$ large enough to guarantee that

$$
\frac{4}{\tau_{q}^{3}}+\frac{4 k \tau_{T}-2 \tau_{q} \tau_{\nu}^{*}}{\tau_{q}^{3} \rho c_{\nu}} \lambda_{n}<0
$$

and then, there exists instability of solutions.

In case that (5.2) holds, but (5.3) fails, we can always select a domain $B$ with a geometry such that the first eigenvalue $\lambda_{1}$ is small enough to guarantee that

$$
\frac{2}{\tau_{q}^{5} \rho c_{\nu}}\left(\tau_{\nu}^{*}-k^{*} \tau_{q}\right)+\frac{\tau_{\nu}^{*} \lambda_{1}}{\tau_{q}^{5} \rho^{2} c_{\nu}^{2}}\left(2 k \tau_{T}-\tau_{\nu}^{*} \tau_{q}\right)<0
$$

Thus, we will obtain unstable solutions to our problem.

We also point out that in case that (5.2) holds and the geometry of $B$ is good enough to guarantee that

$$
\frac{2}{\tau_{q}^{5} \rho c_{\nu}}\left(\tau_{\nu}^{*}-k^{*} \tau_{q}\right)+\frac{\tau_{\nu}^{*} \lambda_{1}}{\tau_{q}^{5} \rho^{2} c_{\nu}^{2}}\left(2 k \tau_{T}-\tau_{\nu}^{*} \tau_{q}\right)>0
$$

then the solutions are stable. 


\section{Conclusion}

In this short note we have analyzed the range of the parameters $\tau_{T}, \tau_{\nu}, k, k^{*}$ and $\tau_{q}$ for the the three-phase-lag theory in order to guarantee the that the solutions of the corresponding heat equation are stable. For the equation (1.1) we have seen that:

1.a If $\tau_{\nu}^{*}-k^{*} \tau_{q}<0$, then there may exist unstable solutions, depending on the domain.

1.b If (3.4) is violated, there always exist unstable solutions.

2. If $\tau_{\nu}^{*}-k^{*} \tau_{q}>0$, then the solutions are always (exponentially) stable.

3 . In the particular case that $k^{*}=0$ the solutions are always stable.

For the equation (1.2) we have seen that:

4. If $\tau_{q} \tau_{\nu}^{*}>2 k \tau_{T}$ there always exist unstable solutions.

5.a If $\tau_{q} \tau_{\nu}^{*}<2 k \tau_{T}$, but $\tau_{\nu}^{*}>k^{*} \tau_{q}$ then there may exist unstable solutions, depending on the domain.

5.b If (5.4) holds, there always exist unstable solutions.

6. If $k^{*} \tau_{q}<\tau_{\nu}^{*}<\frac{2 k \tau_{T}}{\tau_{q}}$, then the solutions are always (exponentially) stable.

7. In the particular case that $k^{*}=0$ the solutions are stable (unstable)

whenever $\tau_{\nu}^{*}<(>) \frac{2 k \tau_{T}}{\tau_{q}}$.

\section{References}

[1] D.S. Chandrasekharaiah, Hyperbolic thermoelasticity: A review of recent literature, Appl. Mech. Rev., 51(1998), pp. 705-729.

[2] H.D. Fernández Sare and R. Racke, On the stability of damped Timoshenko systems - Cattaneo versus Fourier law, Konstanzer Schriften Math. Inf. (2007).

[3] I. Müller and T. Ruggeri, Rational and Extended Thermodynamics, SpringerVerlag, New-York, 1998.

[4] R. Quintanilla, Exponential stability in the dual-phase-lag heat conduction theory, J.Non-Equilibrium Thermodynamics, 27(2002), pp. 217-227.

[5] R. Quintanilla, A condition on the delay parameters in the one-dimensional dualphase-lag thermoelastic theory, J. Thermal Stresses, 26(2003), pp. 713-721.

[6] R. Quintanilla and R. Racke, A note on stability of dual-phase-lag heat conduction. International Journal of Heat and Mass Transfer, 49(2006), pp. 1209-1213.

[7] R. Quintanilla and R. Racke . Qualitative aspects in dual-phase-lag thermoelasticity. SIAM Journal of Applied Mathematics. 66(2006), pp. 977-1001. 
[8] S.K. Roy Choudhuri, On a thermoelastic three-phase-lag model. J. Thermal Stresses, 30(2007), pp.231-238.

[9] D. Y. Tzou, A unified approach for heat conduction from macro to micro-scales, ASME J. Heat Transfer, 117(1995), pp. 8-16.

[10] D. Y. Tzou, The generalized lagging response in small-scale and high-rate heating, Int. J. Heat Mass Transfer, 38(1995), pp. 3231-3240.

[11] L. Wang, M. Mingtian and X. Zhou, Well-posedness and solution structure of dualphase-lagging heat conduction, Int. J. Heat Mass Transfer, 44(2001), pp. 1659-1669.

[12] L. Wang and M. Mingtian, Well-posedness of dual-phase-lagging heat equation: higher dimensions, Int. J. Heat Mass Transfer, 45(2002), pp. 1165-1171.

[13] M. Mingtian and L. Wang, Thermal oscillation and resonance in dual-phase-lagging heat conduction, Int. J. Heat Mass Transfer, 45(2002), pp. 1055-1061. 